\title{
Association of TERT gene polymorphisms with clinical benign prostatic hyperplasia in a Chinese Han population of the Northwest region
}

\author{
Guangrui Fan ${ }^{1 \#}$, Kun Li ${ }^{1 \#}$, Yangyang Pang ${ }^{1}$, Youli Zhao ${ }^{1}$, Yan Tao ${ }^{1}$, Huimin Gui ${ }^{1}$, Hanzhang Wang ${ }^{2}$, \\ Robert Svatek $^{2}$, Ronald Rodriguez ${ }^{2}$, Zhiping Wang ${ }^{1 \wedge}$ \\ ${ }^{1}$ Institute of Urology, Lanzhou University Second Hospital, Key Laboratory of Urological Diseases in Gansu Province, Gansu Nephron-Urological \\ Clinical Center, Lanzhou, China; ${ }^{2}$ Department of Urology, The University of Texas Health Science Center at San Antonio, San Antonio, Texas, USA \\ Contributions: (I) Conception and design: G Fan, Z Wang; (II) Administrative support: Z Wang; (III) Provision of study materials or patients: Y \\ Pang, Y Zhao, Y Tao, H Gui; (IV) Collection and assembly of data: G Fan, K Li; (V) Data analysis and interpretation: G Fan, K Li; (VI) Manuscript \\ writing: All authors; (VII) Final approval of manuscript: All authors. \\ \#These authors contributed equally to this work. \\ Correspondence to: Prof. Zhiping Wang. Institute of Urology, Lanzhou University Second Hospital, 82 Cuiying Gate, Lanzhou 730030, China. \\ Email: wangzplzu@163.com.
}

Background: To investigate the association between single nucleotide polymorphisms (rs10078761, rs12696304, rs2853669, rs16847897, rs2736100, rs10069690) of telomerase gene (TERT) and the risk clinical benign prostatic hyperplasia (BPH) in a Chinese Han population of the Northwest region.

Methods: A total of $150 \mathrm{BPH}$ patients and 150 healthy older males from the northwest Chinese Han population were included in this study. The sample size for this unmatched case-control study was estimated by the look-up table method. Meanwhile, the general information and disease data of patients were collected. Age was only collected in healthy control subjects for statistical correction. Genotypes were detected using a multiplex PCR + ligase detection reaction (LDR). Typing results and clinical data were statistically analyzed using multiple linear regression and logistic regression. Pearson correlation was used for Hardy-Weinberg equilibrium.

Results: The included population is in Hardy-Weinberg equilibrium. There was no significant association between SNP and the risk of BPH by correlation analysis. However, 4 haplotypes (TCTGGT, TCTGTC, TGCCTC, and TGTGTC) were identified as risk factors of BPH by haplotype analysis. The SNP rs2853669 is an independent risk factor for smooth muscle type of hyperplasia. Besides, rs2736100, rs10078761, and rs10069690 which are in linkage disequilibrium are associated with the severity of BPH.

Conclusions: Polymorphism of the TERT gene determines the different disease development and pathological manifestations of $\mathrm{BPH}$ in the Chinese Han population the Northwest region.

Keywords: Telomerase (TERT); telomerase; benign prostatic hyperplasia (BPH); single nucleotide polymorphisms

Submitted Jun 26, 2020. Accepted for publication Dec 02, 2020.

doi: $10.21037 /$ tau-20-1032

View this article at: http://dx.doi.org/10.21037/tau-20-1032

^ ORCID: 0000-0002-8140-591X. 


\section{Introduction}

Benign prostatic hyperplasia (BPH) has gradually become one of the world's serious health problems in men. It is believed to be due to aging and an interplay of sex hormones. Together with prostate cancer (PCa), BPH is the most common neoplastic disease in the older male population (1). To date the etiological agents of $\mathrm{BPH}$ are unknown. Although most men develop benign hyperplasia of the prostate with aging, it is difficult to identify those who will develop severe dysuria making it impossible to prevent that situation effectively. The presence of genetic polymorphisms in the human genome offers us the possibility to study these differences. Among the several studies conducted on the polymorphisms of prostatic hyperplasia: sex hormones, inflammation, metabolic syndrome, and other aspects are the main research directions as the causes of BPH. Nevertheless, the current results fail to confidently clarify the aforementioned problems (2-5).

Telomeres are nucleoprotein structures located at both ends of cell chromosomes that protect chromosome ends and prevent DNA loss (6). Telomeres gradually shorten with each cycle of DNA replication, and cannot divide further after telomere shortening reaches $4 \mathrm{k} b \mathrm{~b}$ in somatic cells. This mechanism of telomere shortening has been reported as one of the mechanisms of cancer suppression in the body (7). For cells with proliferative activity such as the hematopoietic stem cells, telomerase is often activated to prevent the shortening of dividing telomeres, as is the case in tumor cells (8). The structure of telomerase includes reverse transcriptase (TERT), template RNA (TERC) as well as Dyskerin accessory protein, of which the structure of reverse transcriptase is highly conserved (9). Earlier studies have shown that TERT has distinct manifestations in different benign prostatic hyperplasia cells (basal cells, luminal cells, and their progenitor cells) as a novel mechanism in BPH (10). And TERT may be a new target for treating of $\mathrm{BPH}$ (10).

Indeed, in clinical practice, it is easy to observe the differences in the proliferative cell types as indicated by the pathological results of different postoperative tissues of $\mathrm{BPH}$ patients. This increases the assumptions of whether the different manifestations between this population mean that the polymorphism of TERT plays a role in causing BPH. Besides, previous studies have confirmed that TERT plays an important role in PCa (11). Furthermore, previous studies have confirmed that some polymorphisms are associated with $\mathrm{PCa}$ (12). Whether the performance of SNPs in BPH is associated with the performance in prostate cancer is indeed rarely demonstrated. Therefore, this study focused on some selected TERT loci to reveal the performance of TERT polymorphisms in different populations suffering from $\mathrm{BPH}$. The study also aimed to reveal the relationship between $\mathrm{BPH}$ and $\mathrm{PCa}$ by comparing the similarities of SNPs and differences in $\mathrm{BPH}$ and $\mathrm{PCa}$.

We present the following article in accordance with the MDAR reporting checklist (available at http://dx.doi. org/10.21037/tau-20-1032).

\section{Methods}

\section{Specimens and data collection}

The sample size for this unmatched case-control study with equal numbers of subjects in the two groups was estimated by the look-up table method. The estimate that is supported by this method is normally around at least 203 subjects in total. We managed to collect a total of 300 subjects without randomization. After a written informed consent was obtained, 150 whole blood samples from patients diagnosed with $\mathrm{BPH}$ as well as 150 whole blood samples from healthy older men who did not have clinical BPH were collected at the Second Hospital of Lanzhou University between 2013 and 2019. All the blood samples were stored at $-80^{\circ} \mathrm{C}$ in the Institute of Urology, the Second Hospital of Lanzhou University.

The diagnostic criteria of clinical BPH were confirmed by two methods: (I) diagnosed with benign prostatic hyperplasia by imaging, digital rectal examination (DRE) found enlarged prostate but no abnormal nodules in the gland, and also meet PSA $<4 \mathrm{ng} / \mathrm{mL}$; (II) if an enlarged prostate is found by imaging and DRE but PSA $\geq 4 \mathrm{ng} / \mathrm{mL}$ or there are suspicious nodules in the gland, it must be confirmed as BPH by pathological examination to rule out prostate cancer. The diagnosis was made by two experienced urologists based on the above criteria, and a third urologist made the discernment in cases of inconsistent judgment from the other 2 urologists.

The control group was screened from an elderly male population who underwent a health examination at the Healthy Examination Center of the Second Hospital of Lanzhou University to exclude clinical BPH. The inclusion criteria set met the following conditions: no enlargement of the prostate on imaging, no nodules in the gland, and normal PSA (PSA $<4 \mathrm{ng} / \mathrm{mL}$ ). 
The exclusion criteria for all subjects were upper urinary tract diseases, urinary system tumors, neurogenic bladder dysfunction, acute prostatitis, urethral stricture, severe systemic diseases, uncontrolled diabetes, history of the lower urinary tract or transurethral surgery, history of cardiovascular disease, history of mental illness, communication disorders or other diseases which may have interfered with the study results.

The general and clinical data of $\mathrm{BPH}$ patients were collected. They included age, past medical history, height, weight, mean arterial pressure (MAP), International Prostate Symptom Score (IPSS), Quality of Life Score (QOL) and Overactive Bladder Symptom Score (OABSS), peak urinary flow rate $(\mathrm{Qmax})$, prostate volume (PV), post-void residual volume (PVR), serum prostate-specific antigen, pathological findings. Also included was the presence of progress data of BPH such as; recurrent hematuria, recurrent urinary tract infections, acute urinary retention, bladder stones, and abnormal renal function. Data were obtained and recorded mainly through questionnaires or searching medical electronic medical records. For the control group, only age was collected as a statistical adjustment variable. PSA was determined by the ELISA protocol in each subject. Amax and residual urine were valued by the uroflowmetry system (ANDROMEDA; LAKM Medical Technology; Co., Ltd.; Beijing, China). PV was calculated by the data measured by transrectal ultrasonography. The evaluation of PVR for all patients was based on transabdominal urinary ultrasound.

According to the research consensus and clinical guidelines (13), each indicator was analyzed independently and grouped according to actual clinical significance. The criteria were as follows: IPSS, $<20 v s$. $\geq 20$; PSA, $<4$ vs. $\geq 4 \mathrm{ng} / \mathrm{mL}$; PV, $<30 v s . \geq 30 \mathrm{~mL}$; PVR, $<50 v s . \geq 50 \mathrm{~mL}$ and Qmax, $<10 v s . \geq 10 \mathrm{~mL} / \mathrm{sec}$. The quality requirements for the overall representativeness of pathological results were as follows. (I) Puncture tissue: the specimen is well-preserved and fixed in neutral formaldehyde, which is prostate tissue, the number of puncture tissues is $\geq 12$, and each needle tissue section is clearly described in detail. (II) Transurethral resection of the prostate (TURP) tissue: the specimen is wellpreserved and fixed in neutral formaldehyde, the tissue was first divided into at least three tissue boxes, and each box was ensured that it contained at least 8 tissues. Then at least 3 gray, rough, and not significantly cauterized tissue strips were picked $(>1 / 3)$ from each box and that the sections of each tissue were clear and carefully described. (III) The diagnosis was performed by more than two full-time pathologists, and the third pathologist reviewed when the opinions were inconsistent. When the type of pathological hyperplasia was counted, the results of each tissue were independently collated, for one case, the final pathological result was the sum of the proliferation types that had occurred in all the tissue strips. Conversely, if there was no certain pathological type in all the tissue strips of this case, there was no such pathological type in the final result.

This study was conducted in accordance with the Declaration of Helsinki (as revised in 2013). The study was approved by the Ethics Committee of Lanzhou University Second Hospital (No. 2019A-187) and informed consent was taken from all the patients.

\section{SNP selection and genotyping}

To investigate the relationship between the SNPs of the telomerase gene and BPH, 6 SNP sites were selected for the present research. The selection of the SNP sites was guide by information gathered from literature and online databases (https://www.ncbi.nlm.nih.gov/snp/). The 6 SNP sites included rs10078761, rs12696304, rs2853669, rs16847897, rs2736100, and rs10069690. All blood samples collected from the patients at the time of recruitment were maintained in anticoagulant blood collection tubes with EDTA. Genomic DNA was extracted from whole blood samples using a Tiangen DNA extraction kit (Tiangen Biotech Co., Ltd.; Beijing, China). The primers and probes were designed by Primer3 online Version 0.4.0 (http:// frodo.wi.mit.edu/) and Oligo Version 6.31 (Molecular Biology Insights Inc., USA). Basic sequences were searched the on NCBI database. Genotyping was performed with multiplex polymerase chain reactions (PCR) and multiplex ligase detection reaction (LDR) system. Technical support for the experiments mentioned above, reagents and instruments required were provided by Shanghai Biowing Applied Biotechnology Co., Ltd. (Shanghai, China). Genotyping was performed by lab technicians following a blind method that had been set up. All experiments were technically repeated at least three times. Technicians were blinded from all the information of this research which was maintained by the authors. Only the sample number was provided to the technologists.

\section{Statistical analysis}

A pre-association analysis which included Hardy-Weinberg Equilibrium (HWE) and Linkage disequilibrium (LD) was performed. HWE was used to verify the genetic stability 
and representativeness of the analyzed population while LD was used to analyze whether the measured SNPs were linked or not. Also, association analysis studies including single SNP correlation analysis as well as haplotype analysis were performed. The HWE test, LD test, haplotype analysis and correlation analysis were performed using SHEsis software (http://analysis2.bio-x.cn/myAnalysis.php) (14) and SPSS 19.0 software (IBM Corp., Armonk, NY, USA). We further assessed the association between each SNP BPH risk using genetic model analysis. The genetic model first used in the analysis was the additive model. However, other models were also used if statistically significant. Linear correlation analysis and logistic regression analysis were used for correlation analysis.

\section{Results}

\section{Participants data}

We collected and analyzed the clinical data of the experimental subjects, and the analysis results (Table 1) were presented as means and standard deviations (SD). The mean age of the control group $(54.15 \pm 3.67)$ was younger than the experimental group $(68.25 \pm 8.00)(\mathrm{P}<0.05)$. Therefore, age was used as a correction factor in subsequent analyses.

\section{Typing results and pre-association analysis}

After experimental verification, the overall detection rate of genotypes of the samples was $98 \%$. Four samples were not detected including 2 in the control group and 2 in the experimental group. All samples including those not detected were included in the calculation and statistics. In the SHEsis software, undetected genotypes are represented as " $0 / 0$ " and are included in the calculation. The heatmap of specific genotypes for all cases are as shown (Figure 1A).

The results of the HWE are shown in Table 2. From the results of the HWE, it was shown that the total population and subpopulation studied were stably inherited populations $(\mathrm{P}>0.05)$. The results of the LD test are shown in Figure 1B, with a total of 3 SNPs (rs2736100, rs10078761, and rs10069690) in linkage status.

\section{Correlation analysis of disease occurrence risk}

We analyzed the association between each SNP and the risk of clinical BPH by contrasting experimental and control groups (Table 2). From the results of the study, no SNP was shown to independently contribute significantly to the risk of clinical BPH. It was further investigated if the SNPs were in linkage disequilibrium. Also, haplotype analysis was done to add up contributions of SNPs. All haplotypes with genotype frequencies $>0.03$ were included in the association analysis study (Table 3). A total of four haplotypes (highlighted by “*”) (TCTGGT, TCTGTC, TGCCTC, and TGTGTC; Allelic order: rs10069690, rs2736100, rs16847897, rs2853669, rs12696304, rs10078761) were found to be associated with the risk of developing clinical BPH.

\section{Correlation analysis of pathological types}

A total of 70 patients had histological confirmation of the diagnosis of BPH by prostate needle biopsy or transurethral resection of the prostate (TURP). To further confirm the results pathological examination was also performed. In 7 of the cases, only smooth muscle hyperplasia was reported, whereas in the other 63 cases there was glandular hyperplasia, fibroplasia and smooth muscle hyperplasia which were shown to exist simultaneously.

According to extra HWE statistical analysis in the 70 subjects with pathological results, this sub-group was shown to be in a stable population $(\mathrm{P}=0.6292)$. Further analysis revealed that $\mathrm{rs} 2853669 \mathrm{~T}>\mathrm{C}$ is an independent positive correlation factor of the single smooth muscle hyperplasia pathological type (OR 6.07, 95\% CI: $1.56-$ 23.64, $\mathrm{P}=0.004)$ (Table 4).

\section{Correlation analysis of disease progression of $\mathrm{BPH}$}

In the present study, the criteria applied for Assessment of Clinical Progression of BPH included: (I) IPSS $\geq 20$; (II) Qmax $\leq 10 \mathrm{~mL} / \mathrm{s}$; (II) more than two times of hematuria (gross hematuria or microscopic hematuria); (IV) more than two times of evidence of urinary tract infection (urine routine bacteria $\geq 103$ or midstream urine culture positive); (V) imaging showed the presence of stones in the bladder, patients with or without urination interruption phenomenon; (VI) acute urinary retention or indwelling catheterization due to acute urinary retention; and (VII) renal impairment.

Further definitions made was that when grouped, cases with two or more conditions at the same time will be considered as more clinically progressive cases. On the other hand, cases with only one or neither of them were considered as fewer progressive cases.

A total of $150 \mathrm{BPH}$ cases were divided into two groups 
Table 1 Basic characters of all subjects

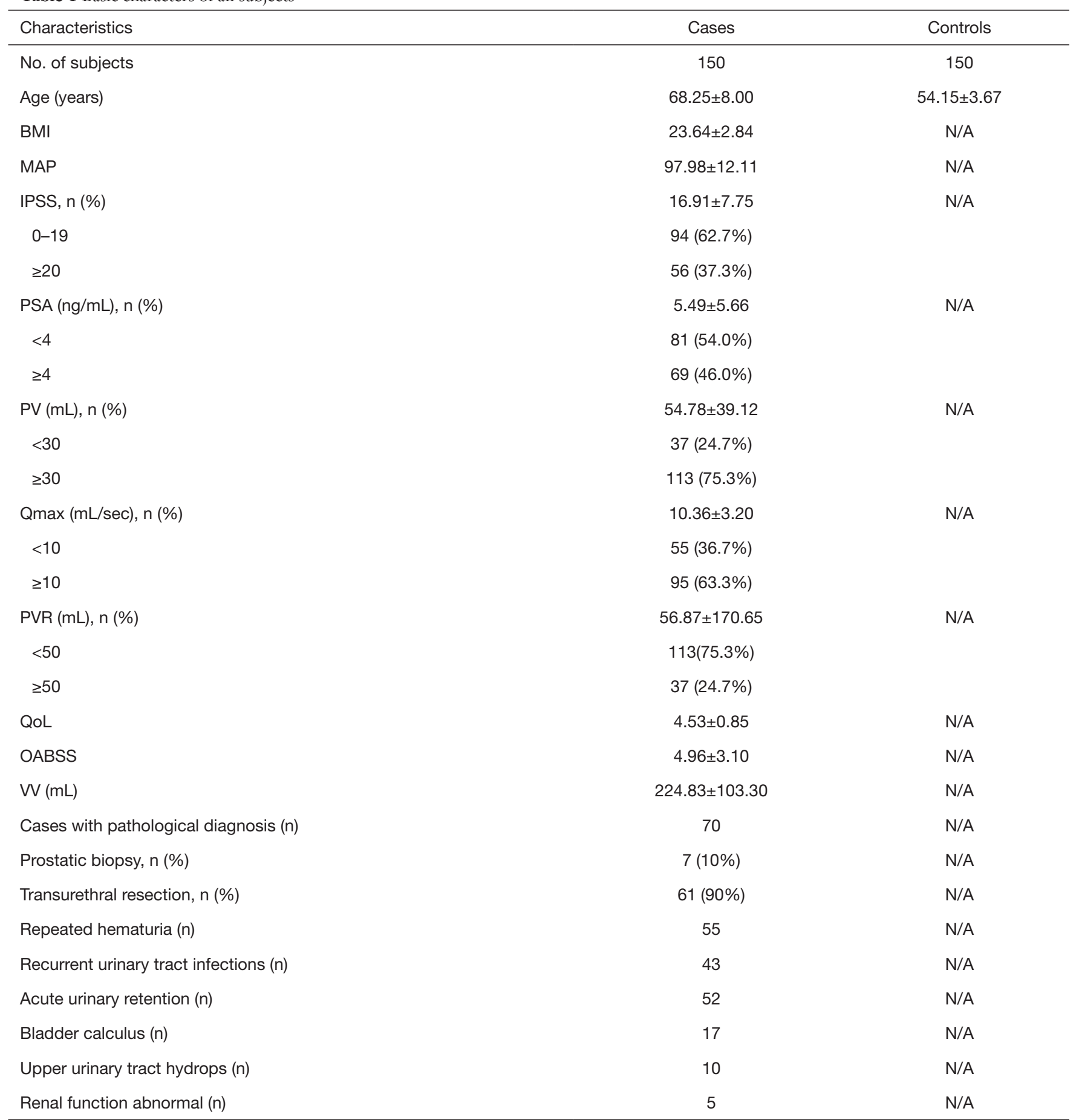

BMI, body mass index; MAP, mean arterial pressure; IPSS, International Prostate Symptom Score; QOL, quality of life score; OABSS, Overactive Bladder Symptom Score; Qmax, peak urinary flowrate; PV, prostate volume; PVR, post-void residual volume; PSA, prostate-specific antigen; VV, voided volume; N/A, not applicable. 
A
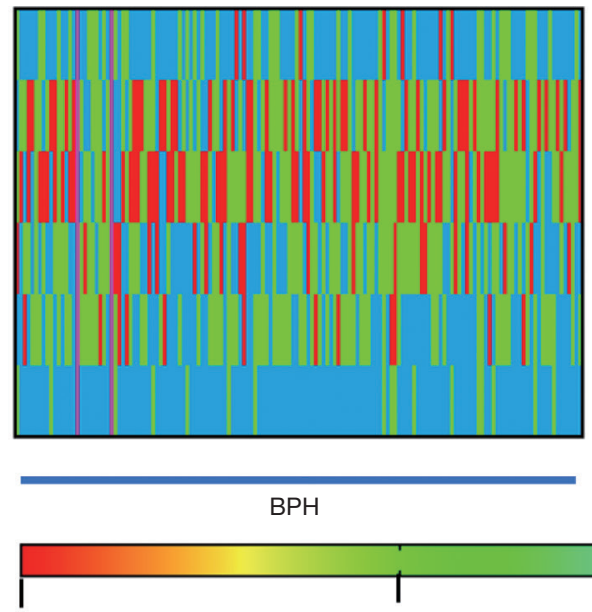

Homozygous Alteration

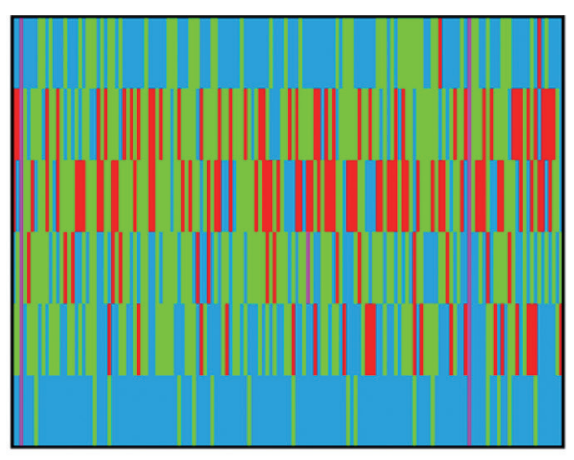

Control

rs10069690

rs2736100

rs16847897

rs2853669

rs12696304

rs10078761

B

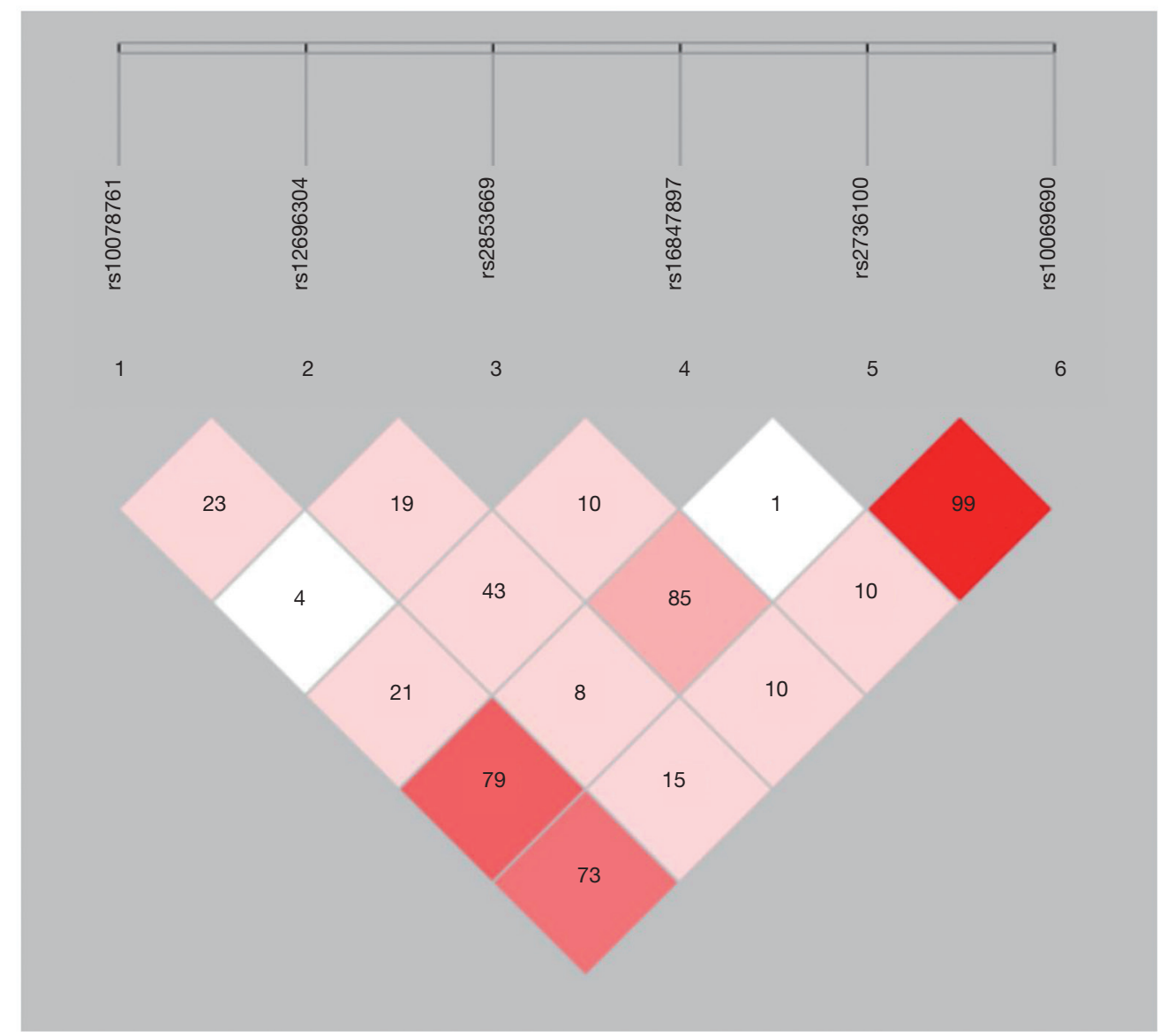

Figure 1 SNPs genotyping and LD analysis. (A) Genotype heatmap of all samples; (B) heatmap of LD analysis between mutation sites. The darker the red block, the higher the Linkage disequilibrium level. 
Table 2 The results of HWE test and correlation analysis of BPH risk

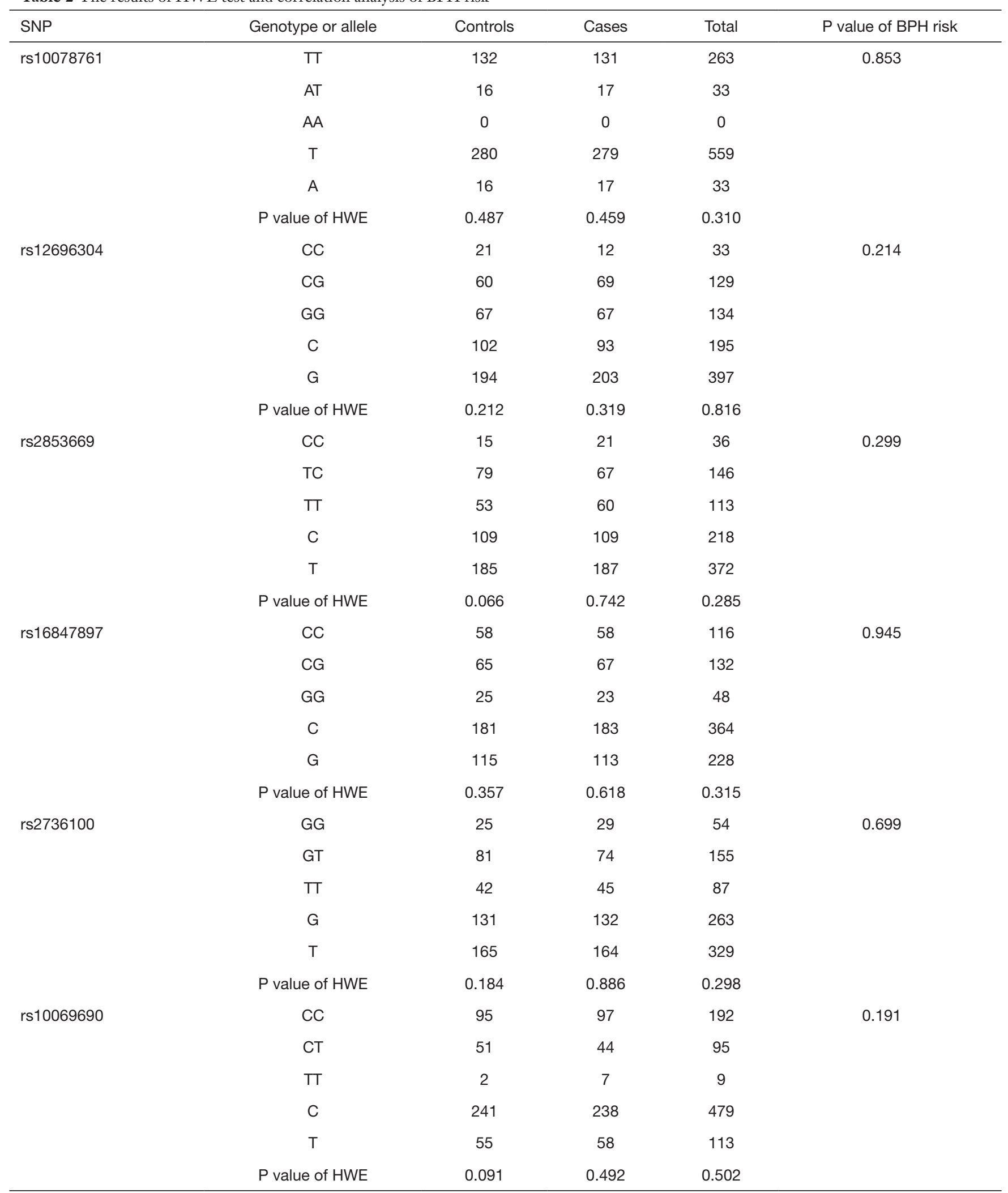

SNP, single nucleotide polymorphism; HWE, Hardy-Weinberg equilibrium. 
Table 3 The results of haplotype analysis

\begin{tabular}{lrrrrrrr}
\hline Haplotype & Case (freq) & Control (freq) & Chi2 & Monte Carlo P & Fisher's P & Pearson's P & Odds ratio (95\% Cl) \\
\hline TCCGGC & $10.21(0.035)$ & $19.39(0.066)$ & 2.909 & 1 & 0.0881 & 0.0881 & $0.513(0.235-1.118)$ \\
TCTCTC & $16.89(0.057)$ & $12.12(0.041)$ & 0.897 & 1 & 0.3437 & 0.3436 & $1.441(0.674-3.082)$ \\
TCTGGT* & $11.38(0.038)$ & $3.55(0.012)$ & 4.335 & 0.8333 & 0.0374 & 0.0373 & $3.354(1.006-11.190)$ \\
TCTGTC* & $25.48(0.086)$ & $46.89(0.159)$ & 7.119 & 0.8333 & 0.0077 & 0.0076 & $0.498(0.296-0.836)$ \\
TGCCGC & $34.89(0.118)$ & $26.60(0.090)$ & 1.400 & 1 & 0.2368 & 0.2367 & $1.383(0.807-2.371)$ \\
TGCCGT & $5.87(0.020)$ & $13.99(0.048)$ & 3.350 & 1 & 0.0673 & 0.0672 & $0.411(0.154-1.096)$ \\
TGCCTC* & $21.88(0.074)$ & $11.10(0.038)$ & 3.923 & 0.8333 & 0.0477 & 0.0476 & $2.094(0.994-4.412)$ \\
TGCGGC & $9.12(0.031)$ & $13.58(0.046)$ & 0.863 & 1 & 0.3529 & 0.3528 & $0.667(0.283-1.574)$ \\
TGTCGT & $14.36(0.049)$ & $18.11(0.062)$ & 0.415 & 1 & 0.5197 & 0.5197 & $0.791(0.386-1.618)$ \\
TGTCTC & $57.89(0.196)$ & $71.72(0.244)$ & 1.778 & 1 & 0.1824 & 0.1823 & $0.760(0.508-1.138)$ \\
TGTGTC* & $35.58(0.120)$ & $9.56(0.033)$ & 16.876 & 0.1667 & $4.05 \mathrm{E}-05$ & $4.03 \mathrm{E}-05$ & $4.241(2.028-8.869)$ \\
Global result & 294.00 & 296.00 & 39.787 & $\mathrm{~N} / \mathrm{A}$ & $1.94 \mathrm{E}-05$ & $1.85 \mathrm{E}-05$ & $\mathrm{~N} / \mathrm{A}$ \\
\hline
\end{tabular}

*, a statistically significant result. SNP, single nucleotide polymorphism; N/A, not applicable.

Table 4 Pathological type association with rs2853669

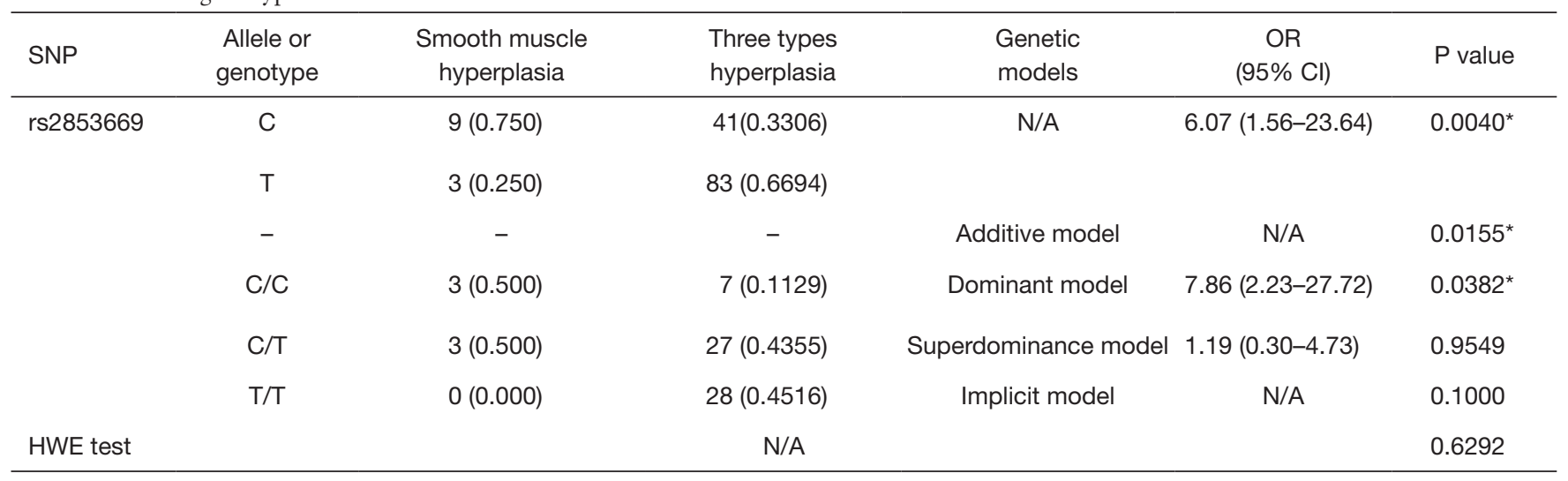

Three types hyperplasia, glandular hyperplasia, fibroplasia, and smooth muscle hyperplasia concurrence. * , a statistically significant result. SNP, single nucleotide polymorphism. HWE, Hardy-Weinberg equilibrium; N/A, not applicable.

according to the above criteria: progressive group and nonprogressive group. After analysis as shown in Table 5, it was established that three SNPs (rs2736100 T>G, rs10078761 $\mathrm{T}>\mathrm{A}$, and $\mathrm{rs} 10069690 \mathrm{C}>\mathrm{T}$ ) were significantly associated with progressive cases. According to the results of the aforementioned linkage disequilibrium test, these three SNPs are linked to each other. Hence, meaning that the linked genotypes composed of the 3 SNPs together are closely related to the clinical progression of $\mathrm{BPH}$.

\section{Discussion}

There is substantial literature demonstrating a correlation between symptomatic BPH and genetic predisposition $(15,16)$. Unfortunately, the polygenic nature and the subjective phenotypic expression of $\mathrm{BPH}$ have made predictive genetic screening impractical. In other words, there are multiple pathways to the end state called $\mathrm{BPH}$, manifesting as urinary outlet obstruction.

$\mathrm{BPH}$ is a proliferative disorder characterized by glandular 
Table 5 The relevance between progressive cases and SNPs

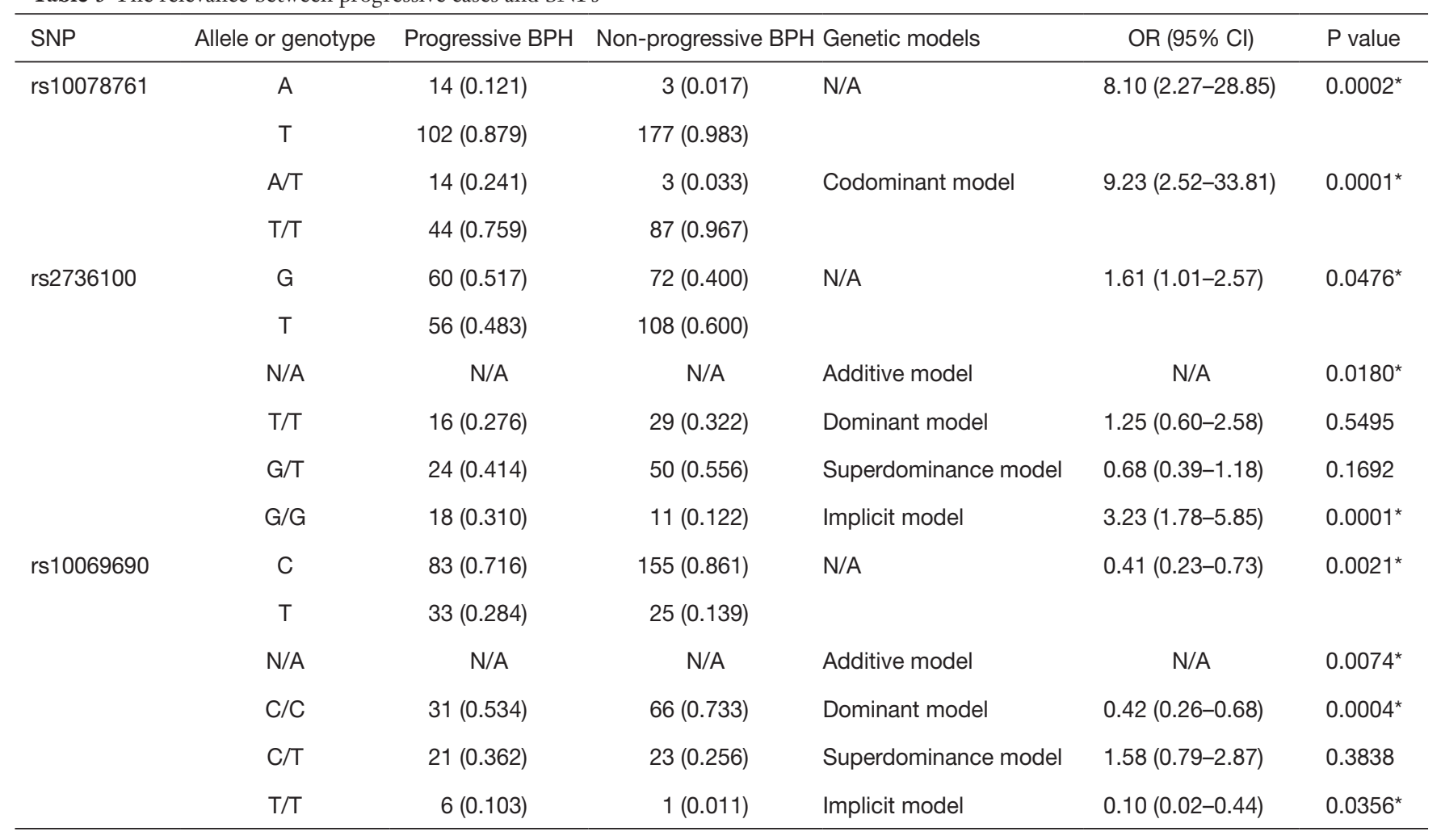

Three types hyperplasia, glandular hyperplasia, fibroplasia, and smooth muscle hyperplasia concurrence. * , a statistically significant result. SNP, single nucleotide polymorphism. HWE, Hardy-Weinberg equilibrium; N/A, not applicable.

or smooth muscle proliferation, with some neoplastic properties. Although most scientists believe that $\mathrm{BPH}$ and prostate cancer are independent disease entities and one does not lead to the other, some researchers have suggested some similarities between $\mathrm{BPH}$ and prostate cancer (PCa) $(17,18)$, as they both share loss of contact growth inhibition. From previous studies, it has been reported that one of the earliest gene expression changes required for dysregulated growth inhibition, is the induction and maintenance of telomerase. Telomere length has been shown to be closely related to the status and prognosis of $\mathrm{PCa}(19,20)$. However, the relationship between telomere length and the risk of PCa is controversial $(21,22)$. In addition, it has been found that committed basal cells have shorter telomeres than luminal cells in BPH (23), which not only means that the progenitor cells from which the two type of cells are derived are not the same, but may also have different telomerase status during differentiation and maturation. In the present study, it was found that cases of smooth muscle hyperplasia were affected by the rs2853669 C allele than cases of mixed glandular and smooth muscle hyperplasia. The rs2853669 mutation has been shown to enhance the susceptibility to $\mathrm{PCa}$ (24). Therefore, based on our findings it can be further speculated that $\mathrm{BPH}$ with predominant smooth muscle hyperplasia caused by rs2853669 may share common pathways of activation to PCa.

Several studies have shown that the SNPs selected for the present have an important status in a variety of solid or hematological tumors $(12,25)$. Previous studies have found these 3 SNPs; rs2736100, rs10078761, and rs10069690 to be associated with prostate cancer (26). In our study, these three SNPs happened to be associated with the severity of outlet obstruction from $\mathrm{BPH}$ and had linkage disequilibrium relationships by single-locus analysis and linkage disequilibrium test. These results indicate that these three SNPs cluster together, and correlate with severe signs \& symptoms of BPH. Similarly, according to the relationship between rs2736100, rs10078761, rs10069690 and $\mathrm{PCa}$, we can speculate that the existence of this cluster may share pathways of TERT activation between PCa and symptomatic $\mathrm{BPH}$.

Recently, there has been great progress in the 
development of sequencing technology. Hence it is no longer a major problem to explore the genetic information of human beings. Without the completion and application of the Human Genome Project (HGP), the Genome-wide association study (GWAS) and the haplotype mapping (HapMap), it is possible to understand the contribution of different phenotypes within genes to diseases. Results from many studies have shown that when the strength of an individual SNP effect is limited, haplotypes which can be seen as additive effects are a better way to explain disease's phenomena (27). In our research, although no single SNP was shows to make an independent contribution to the pathogenesis of $\mathrm{BPH}$, we found that a panel of SNPs (TCTGGT, TCTGTC, TGCCTC, and TGTGTC) was correlated with the risk of BPH. However, it should be noted that the results found are only preliminary, and further confirmation of whole haplotypes is required due to the limitation of the number of sites used in the present study. What we can confirm, however, is that haplotypebased research protocols are a valuable approach to the study of symptomatic BPH development. This approach may then, unmask various subtypes of BPH based on molecular profiles (e.g., smooth muscle versus glandular hyperproliferation). In addition, although we used statistical methods to minimize the effect of age between different groups, due to the strong correlation between $\mathrm{BPH}$ onset and age, this study may still have the bias of incidence between different groups. We believe that a more meticulous and rigorous study grouping design would be helpful in reducing such bias.

\section{Conclusions}

In conclusion, the results of this research suggest that the $\mathrm{C}$ allele of rs2853669 is an independent risk factor for smooth muscle type of hyperplasia, and the linked rs2736100, rs10078761 and rs10069690 increase the risk of symptomatic BPH together. We also identified a total of four haplotypes (TCTGGT, TCTGTC, TGCCTC, and TGTGTC) which are associated with the development of BPH. The evidence from the current study leads us to believe that the polymorphism of the TERT gene determines the different disease development and pathological manifestations of $\mathrm{BPH}$ in the population we mentioned.

\section{Acknowledgments}

Funding: This study was funded by the National Natural Science Foundation of China (81874088), the Cuiying Technology
Innovation Program of Lanzhou University Second Hospital (CY2017-MS01) and the Fundamental Research Funds for the Central Universities (lzujbky-2018-kb14).

\section{Footnote}

Reporting Checklist: The authors have completed the MDAR reporting checklist. Available at http://dx.doi.org/10.21037/ tau-20-1032

Data Sharing Statement: Available at http://dx.doi. org/10.21037/tau-20-1032

Conflicts of Interest: All authors have completed the ICMJE uniform disclosure form (available at http://dx.doi. org/10.21037/tau-20-1032). The authors have no conflicts of interest to declare.

Ethical Statement: The authors are accountable for all aspects of the work in ensuring that questions related to the accuracy or integrity of any part of the work are appropriately investigated and resolved. The study was conducted in accordance with the Declaration of Helsinki (as revised in 2013). The study was approved by the Ethics Committee of Lanzhou University Second Hospital (No. 2019A-187) and informed consent was taken from all the patients.

Open Access Statement: This is an Open Access article distributed in accordance with the Creative Commons Attribution-NonCommercial-NoDerivs 4.0 International License (CC BY-NC-ND 4.0), which permits the noncommercial replication and distribution of the article with the strict proviso that no changes or edits are made and the original work is properly cited (including links to both the formal publication through the relevant DOI and the license). See: https://creativecommons.org/licenses/by-nc-nd/4.0/.

\section{References}

1. DeSantis CE, Miller KD, Dale W, et al. Cancer statistics for adults aged 85 years and older, 2019. CA Cancer J Clin 2019;69:452-67.

2. Gu X, Na R, Huang T, et al. SRD5A1 and SRD5A2 are associated with treatment for benign prostatic hyperplasia with the combination of 5alpha-reductase inhibitors and alpha-adrenergic receptor antagonists. J Urol 2013;190:615-9. 
3. Yoo KH, Kim SK, Chung JH, et al. Nitric oxide synthase 2 gene polymorphisms are associated with prostatic volume in Korean men with benign prostatic hyperplasia. Asian J Androl 2010;12:690-6.

4. Kwon H, Kang HC, Lee JH. Relationship Between Predictors of the Risk of Clinical Progression of Benign Prostatic Hyperplasia and Metabolic Syndrome in Men With Moderate to Severe Lower Urinary Tract Symptoms. Urology 2013;81:1325-9.

5. Pang Y, Li H, Gong Y, et al. Association of CCL2, CCR2 and CCL5 genetic polymorphisms with the development and progression of benign prostatic hyperplasia. Oncol Rep 2019;41:2491-501.

6. Blasco MA. Telomere length, stem cells and aging. Nature chemical biology 2007;3:640-9.

7. Herrmann M, Pusceddu I, März W, et al. Telomere biology and age-related diseases. Clin Chem Lab Med 2018;56:1210-22.

8. Pestana A, Vinagre J, Sobrinho-Simoes M, et al. TERT biology and function in cancer: beyond immortalisation. J Mol Endocrinol 2017;58:R129-r46.

9. Blackburn EH. Telomeres and telomerase: the means to the end (Nobel lecture). Angewandte Chemie 2010;49:7405-21.

10. Pickard A, Mills IG. Maintaining a Healthy Balance: Targeting TERT to Stem Benign Prostatic Hyperplasia. Eur Urol 2016;69:555-6.

11. Graham MK, Meeker A. Telomeres and telomerase in prostate cancer development and therapy. Nature reviews Urology 2017;14:607-19.

12. He G, Song T, Zhang Y, et al. TERT rs 10069690 polymorphism and cancers risk: A meta-analysis. Mol Genet Genomic Med 2019;7:e00903.

13. Gratzke C, Bachmann A, Descazeaud A, et al. EAU Guidelines on the Assessment of Non-neurogenic Male Lower Urinary Tract Symptoms including Benign Prostatic Obstruction. Eur Urol 2015;67:1099-109.

14. Shi YY, He L. SHEsis, a powerful software platform for analyses of linkage disequilibrium, haplotype construction, and genetic association at polymorphism loci. Cell Res 2005;15:97-8.

15. Sanda MG, Beaty TH, Stutzman RE, et al. Genetic susceptibility of benign prostatic hyperplasia. J Urol 1994;152:115-9.

16. Cartwright R, Mangera A, Tikkinen KAO, et al. Systematic review and meta-analysis of candidate gene association studies of lower urinary tract symptoms in men. Eur Urol 2014;66:752-68.

17. Tayeb MT, Clark C, Haites NE, et al. CYP3A4 and
VDR gene polymorphisms and the risk of prostate cancer in men with benign prostate hyperplasia. Br J Cancer 2003;88:928-32.

18. Tayeb MT, Clark C, Haites NE, et al. Vitamin D receptor, HER-2 polymorphisms and risk of prostate cancer in men with benign prostate hyperplasia. Saudi Med J 2004;25:447-51.

19. Heaphy CM, Gaonkar G, Peskoe SB, et al. Prostate stromal cell telomere shortening is associated with risk of prostate cancer in the placebo arm of the Prostate Cancer Prevention Trial. Prostate 2015;75:1160-6.

20. Jia H, Wang Z. Telomere Length as a Prognostic Factor for Overall Survival in Colorectal Cancer Patients. Cell Physiol Biochem 2016;38:122-8.

21. Wentzensen IM, Mirabello L, Pfeiffer RM, et al. The association of telomere length and cancer: a meta-analysis. Cancer Epidemiol Biomarkers Prev 2011;20:1238-50.

22. Zhang X, Zhao Q, Zhu W, et al. The Association of Telomere Length in Peripheral Blood Cells with Cancer Risk: A Systematic Review and Meta-analysis of Prospective Studies. Cancer Epidemiol Biomarkers Prev 2017;26:1381-90.

23. Rane JK, Greener S, Frame FM, et al. Telomerase Activity and Telomere Length in Human Benign Prostatic Hyperplasia Stem-like Cells and Their Progeny Implies the Existence of Distinct Basal and Luminal Cell Lineages. Eur Urol 2016;69:551-4.

24. Shadrina AS, Boyarskikh UA, Oskina NA, et al. TERT polymorphisms rs2853669 and rs7726159 influence on prostate cancer risk in Russian population. Tumour Biol 2015;36:841-7.

25. Shen N, Lu Y, Wang X, et al. Association between rs2853669 in TERT gene and the risk and prognosis of human cancer: a systematic review and meta-analysis. Oncotarget 2017;8:50864-72.

26. Wu D, Yu H, Sun J, et al. Association of genetic polymorphisms in the telomerase reverse transcriptase gene with prostate cancer aggressiveness. Mol Med Rep 2015;12:489-97.

27. International HapMap Consortium. The International HapMap Project. Nature 2003;426:789-96.

Cite this article as: Fan G, Li K, Pang Y, Zhao Y, Tao Y, Gui H, Wang H, Svatek R, Rodriguez R, Wang Z. Association of TERT gene polymorphisms with clinical benign prostatic hyperplasia in a Chinese Han population of the Northwest region. Transl Androl Urol 2021;10(2):692-702. doi: 10.21037/ tau-20-1032 